\title{
3D Reconstruction of a Human Body from Multiple Viewpoints
}

\author{
Koichiro Yamauchi, Hideto Kameshima, Hideo Saito, and Yukio Sato \\ Graduate School of Science and Technology, Keio University \\ Yokohama 223-8522, Japan \\ \{yamauchi, kameshima, saito\} @ozawa.ics.keio.ac.jp, \\ satoy@ics.keio.ac.jp
}

\begin{abstract}
A human body measurement system using multiple viewpoints is proposed. Whole human body data taken from systems, which have been developed with a few viewpoints, have not been successful at acquiring due to occlusion. It is proposed that data can be successfully obtained by a method using multiple rangefinders correctly. Four compact rangefinders are installed in a pole. Those three pole units, with 12 rangefinders, are assigned around a person. Multiple viewpoint range images allow the 3D shape reconstruction of a human body. Then a morphable model is adapted to whole human body data. The measurement time is 2 seconds and the average error is found to be $1.88 \mathrm{~mm}$. In this paper, the system configuration, calibration, morphable model and experimental results are described.
\end{abstract}

Keywords: human body, 3d reconstruction, modeling, rangefinder.

\section{Introduction}

A rangefinder acquires the $3 \mathrm{D}$ shape (i.e. a range image and a surface image) of a target object by observing it. It has been a suitable device for practical use thanks to the continual improving accuracy, miniaturization and price reduction. Recently, human body measurement has attracted the attention of the research and the business fields. For example whole human body data is applicable to animate digital human models by using motion capture data, based on a prediction model or kinematics. Other applications are the health management, surgical simulation, augmented reality, computer-aided design (CAD), and custom-made clothing.

Some human measurement systems have already been developed and have reached the commercial market. One such product is the Whole Body Color 3D Scanner by Cyberware [1. The measurement time for measuring instruments to move down from a head to toe is 17 seconds. VITUS 3D Body Scanner is a product of VITRONIC [2. It is based on the light-section method, acquiring whole human body data with a resolution of $4-5 \mathrm{~mm}$ in 12 seconds. Another product, which is composed of four range sensors, measures only from the front and the rear 3. Projectors emit structured light patterns in turn and respective cameras

D. Mery and L. Rueda (Eds.): PSIVT 2007, LNCS 4872, pp. 439 448, 2007.

(C) Springer-Verlag Berlin Heidelberg 2007 


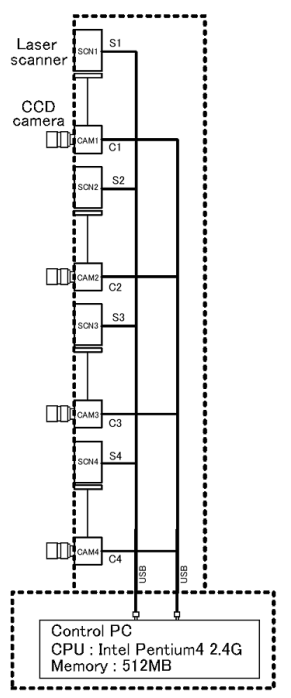

Fig. 1. Configuration of pole unit

capture images of those views. The measurement time is about 8 seconds. Because there are only a few viewpoints, a person must stand a little further away and occlusion will also occur. Although these systems are useful for accuracy and resolution, it takes a long time to obtain whole human body data.

In contrast, stereo methods for 3D shape reconstruction with multiple viewpoints have already been presented. A pertinent instance is the "3D Room" which has 49 cameras are mounted inside the room [4], 5], 6]. Virtual views are generated by model-based rendering and image-based rendering. It is a large facility for digitizing dynamic events.

Generally speaking, compact 3D human measurement systems acquiring a number of accurate data points are desired. There are some important problems we should resolve. First one of them is the measurement time. It is very hard for persons to keep standing motionless during the measurement. In addition, they move increasingly every second. Therefore, we must complete the measurement in the shortest possible time. Another problem is occlusion which is the hidden regions from the views. When we utilize only a couple of viewpoints, occlusion will occur easily. It is especially difficult to capture data of a submental, an axillary, or a groin region, which are often hidden from the views. Therefore, it is better to obtain whole human body data using multiple rangefinders.

In this paper, we use appropriately positioned multiple rangefinders to resolve the previous problems. We have developed a compact rangefinder and installed four rangefinders in a pole. The human body measurement system is configured with three poles in compact space. Multiple range images allow the 3D shape reconstruction of a human body at high speed. Then a morphable model is adapted to the whole human body data. In the following sections, the system configuration, calibration, morphable model and experimental results are described. 


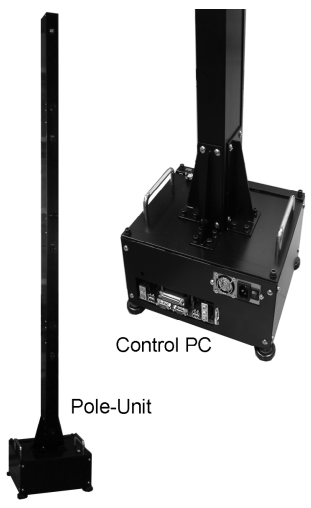

Fig. 2. Pole unit
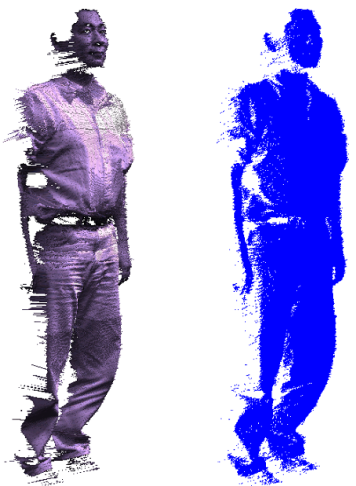

Fig. 3. Measurement result by pole unit

\section{Human Body Measurement System}

\section{$2.1 \quad$ Pole Unit}

The compact rangefinder that we have developed is composed of a CCD camera and a laser scanner 7]. The CCD camera captures an image at $640 \times 480$ pixels resolution with $6 \mathrm{~mm}$ lens. The light source of the laser scanner is red-color semiconductor laser with $650 \mathrm{~nm}$ wavelength. A light pattern is generated from emitting and switching of a slit light made by the laser scanner. The range image is obtained by the space encoding method within 0.5 seconds [8], 9]. 3D coordinate points are computed by the triangulation principle. When we measure a target placed one meter ahead, the measurement error is found to be $2 \mathrm{~mm}$.

Four compact rangefinders are installed in a pole as shown Fig. 1. And, Fig. 2 shows the pole unit and the control PC. It is an independent and movable unit. The size is $300 \mathrm{~mm}$ wide, $265 \mathrm{~mm}$ long and $2135 \mathrm{~mm}$ high. The CCD camera is placed under the laser scanner in each rangefinder. A baseline is $330 \mathrm{~mm}$ between the CCD camera and the laser scanner. The pole unit makes measuring range infinitely wider than one rangefinder. When we measure a target placed one meter ahead, measuring range will be $800 \times 1800 \mathrm{~mm}$. Each rangefinder is connected with the control PC by a USB2.0 cable. The control PC takes control of each rangefinder and synchronizes actions of four rangefinders. Fig. 3 is a measurement result by one pole unit when we have measured a man from the front. The left is texture display and the right is point cloud display. We have obtained the 3D shape of a human body from head to toes. Whole human body data is acquired by using more than one pole unit.

\subsection{System Configuration}

When we measure human bodies, it is difficult to capture data of the submental, axillary and groin regions. If there are a few viewpoints, occlusion will be 


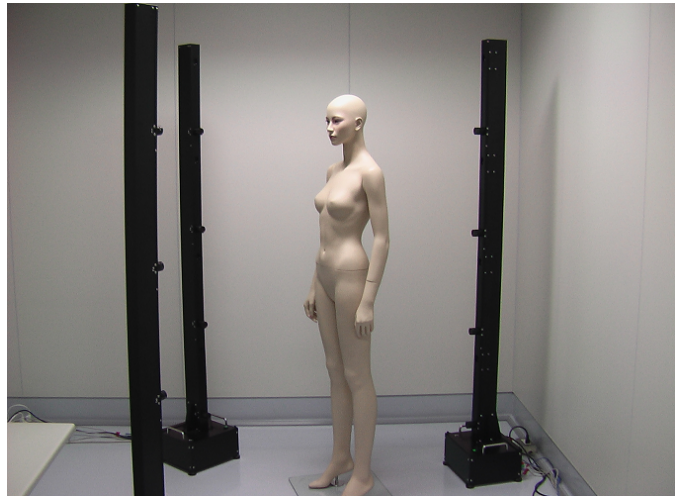

Fig. 4. Human body measurement system

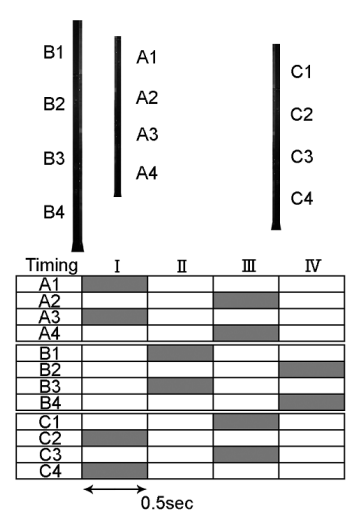

Fig. 5. Timing diagram

found easily. The measuring range of one rangefinder is narrow. Therefore, these problems are solved by using multiple rangefinders.

Three pole units, with 12 rangefinders, are assigned around a human. Fig. 4 is a scene of the measurement. 12 viewpoint range images allow the $3 \mathrm{D}$ shape reconstruction of a human body. Whole human body data, 1.5 million point cloud and 12 surface textures, is obtained.

We must complete the measurement as quickly as possible, preferably within one second, because it is hard to let a person keep standing motionless. If 12 rangefinders of three pole units are sequentially operated one by one, the measurement time is too long. Furthermore, if some rangefinders are performed concurrently, coarse whole human body data will be generated because of light pattern interferences among rangefinders. This adverse effect is suppressed by a combination and a control of the measurement timing. We acquire whole human body data at four times. Fig. 5 is the timing diagram. Four rangefinders placed in a diagonal corner or noninterference height are operated at the same time. It is equal to the measurement time of one rangefinder. Therefore, the measurement time is 2 seconds at four times.

It is possible to improve the accuracy by increasing the number of pole units and conversely to make system more compact with two pole units. The assignment of three pole units is not fixed and able to move itself flexibly.

\section{Calibration}

A simple calibration is performed for the rangefinder. Our rangefinder is composed of the CCD camera and the laser scanner. Camera parameters are the focal length, image centre, skew, and coefficients of the radial distortion. Scanner parameters are the projection angles and baseline between the optical center of the camera and the light source of the scanner. We execute Tsai's camera calibration program to acquire camera parameters [10]. Scanner parameters are obtained using theoretical figures of its design. Two parameter set enable the 


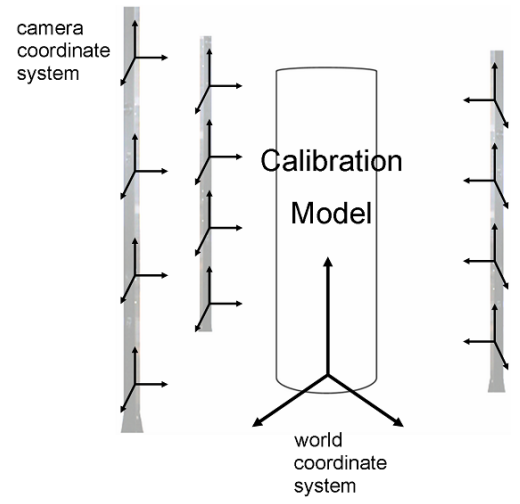

Fig. 6. Calibration model

rangefinder to capture a range image and a surface image of a target object. 3D coordinates are computed by the triangulation principle.

The human body measurement system is configured with three pole units. If geometric positions of rangefinders are not known, 12 range images can not allow the $3 \mathrm{D}$ shape reconstruction of a human body. Our solution to this problem is an alignment approach using a calibration target known 3D shape, such as a cuboid and a cylinder. Calibration model is shown in Fig. 6. The camera coordinate systems of 12 rangefinders are integrated into the world coordinate system of the calibration target. 3D measurement point from the rangefinder is denoted by $\tilde{c}=\left[x_{c}, y_{c}, z_{c}, 1\right]^{T}$. 3D calibration point on the calibration target is denoted by $\tilde{w}=\left[x_{w}, y_{w}, z_{w}, 1\right]^{T}$. The relationship between the camera coordinate system and the world coordinate system is given by

$$
\tilde{w}=\mathbf{H}_{e} \tilde{c} \quad \text { with } \mathbf{H}_{e}=\left[\begin{array}{cccc}
r_{11} & r_{12} & r_{13} & t_{1} \\
r_{21} & r_{22} & r_{23} & t_{2} \\
r_{31} & r_{32} & r_{33} & t_{3} \\
0 & 0 & 0 & 1
\end{array}\right]
$$

where $\mathbf{H}_{e}$ is the Euclidean transformation; 12 parameters $\left[r_{11}, \ldots, t_{3}\right]^{T}$ are solved by the least square method. When 12 Euclidean transformation matrix set are obtained, 12 range images can be integrated into whole human body data. The assignment of pole units has no constraint as long as all rangefinders can observe the calibration target.

\section{Morphable Model}

Many researchers are studying for the 3D modeling of human bodies. For example, the Stanford Digital Michelangelo Project [11, 12] is famous for protecting 3D graphics content. B. Allen et al. [13, [14] proposed a method for creating a whole-body morphable model based on 3D scanned examples. 


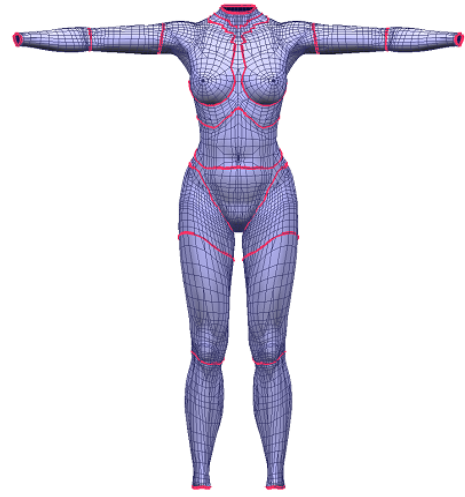

(a) female

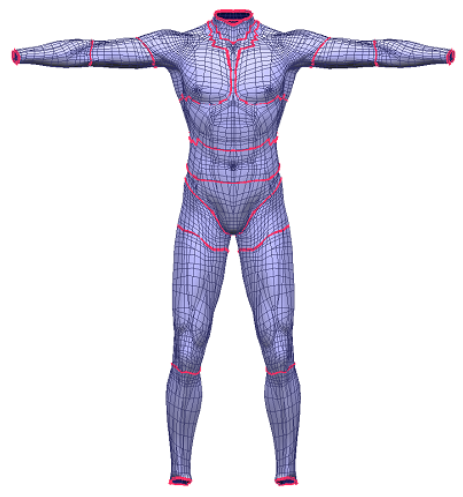

(b) male

Fig. 7. Morphable models

In this section, a modeling technique using whole human body data, which is obtained by multiple rangefinders, is presented. We consider the problems involved with bodily habitus and absent parts. A human body is known more commonly as a closed surface, which declares itself as the same topological object. It is necessary to emphasize that few 3D human body models have the capacity to represent the figure of all persons. Therefore, we have designed two 3D human body models based on Poser 5.0 [15] figures for representation of human bodies.

A 3D female model (7,973 vertices and 8,409 faces) and a 3D male model (8,994 vertices and 8,582 faces) are generated as shown in Fig. 7. Twenty feature curves are defined in these models as closed curves which indicate the boundaries of some body parts. Whole human body data is associable with other obtained data using 3D human body models including region information.

A human body is treated as a rigid object, but our models are treated as an elastic object like a wetsuit. To adapt the proposed 3D model to whole human body data, the mass and-spring-based fitting technique is utilized for deforming elastic objects [16]. The operator is able to handle some feature curves interactively so that whole human body data is wrapped around the proposed model. In addition, the operator can adjust mismatched parts of the adapted model along the surface shape interactively. Consequently, the 3D human body model can be adapted to whole human body data as if it had been dressed in a wetsuit. The adapted model is useful for various applications thanks to the region information.

\section{Experimental Results}

\subsection{Calibration}

A cylinder known 3D shape (415 mm diameter and $1800 \mathrm{~mm}$ height) is utilized for calibration. It is placed in the center of the human body measurement system. This cylinder shape roughly equals to a standard human body and avails 


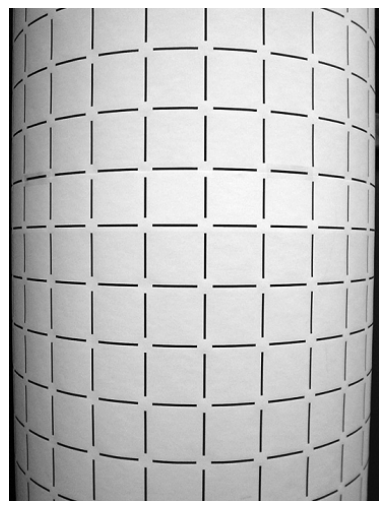

Fig. 8. Calibration cylinder

Table 1. Measurement accuracy

\begin{tabular}{cc}
\hline The number of points & 33 \\
\hline Average error $[\mathrm{mm}]$ & 1.88 \\
\hline Standard deviation $[\mathrm{mm}]$ & 0.79 \\
\hline
\end{tabular}

the improvement of the calibration precision. A lattice pattern is drawn on the surface of the cylinder as shown in Fig. 8. The intersection of a row and column bar is defined as a calibration point. We utilize 80 calibration points or more for the calibration.

The measurement error is searched by using two rangefinders. When two rangefinders measure a same calibration point, one and the other $3 \mathrm{D}$ coordinate are denoted by $\mathbf{p}_{i}$ and $\mathbf{p}_{i}^{\prime}$. The measurement error is defined by

$$
\text { Error }=\frac{1}{N} \sum_{i=1}^{N}\left\|\mathbf{p}_{i}-\mathbf{p}_{i}^{\prime}\right\|
$$

Euclidean distance between $\mathbf{p}_{i}$ to $\mathbf{p}_{i}^{\prime}$; Table. 1 shows the average error and the standard deviation. 33 calibration points are used in this evaluation. This result is within 0.2 percent of the distance to a target.

\subsection{Measurement and Modeling}

We have measured a female mannequin (Fig. 9), a male (Fig. 10), and a clothed male (Fig. 11). Measurement data is displayed in front and back views. Whole human body data is successfully acquired, especially at the submetal, axillary and groin regions. Wrinkles in a fabric also can be obtained. The head hair shape has only few 3D points due to low specular reflectivity for brunet hair. We know that a person moves increasingly second by second. Because the proposed system completes the measurement so quickly, it has little effect. 

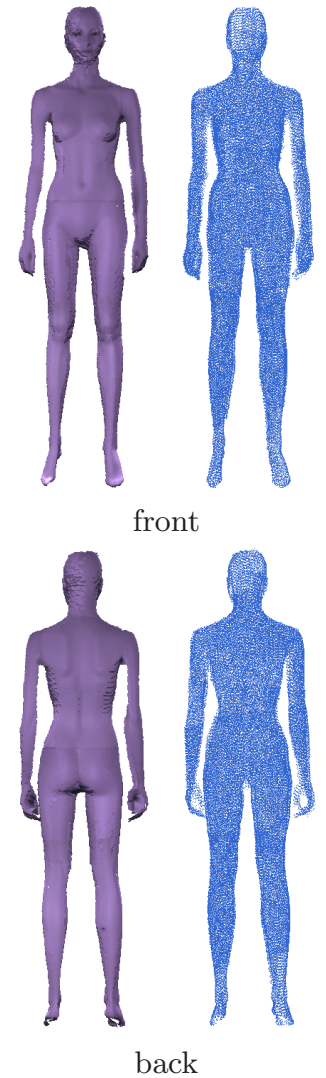

Fig. 9. Measurement result of a female
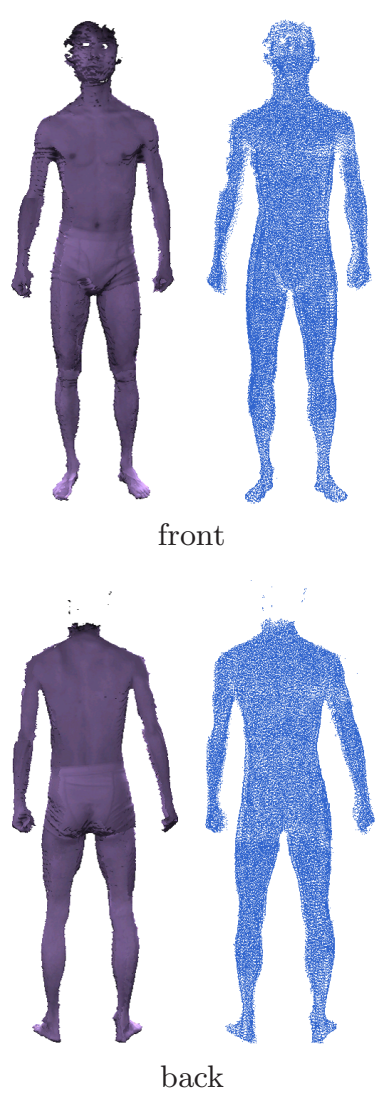

Fig. 10. Measurement result of a male

Experimental results show occlusion problem is solved by multiple rangefinders, but the dead space in the side of a body is caused by long vertical bodily habitus. Then the morphable models are adapted to measurement data of a female and a male. Fig. 12 and Fig. 13 show modeling results of a female and a male, respectively. Because proposed morphable models are closed surfaces, some holes and absent parts are automatically covered. Using region information (the positions of arms, elbows, knees and so forth), the adapted model will be utilized for various applications. At the present stage, basic motions, such as walking or running, have been realized [17].

\section{Conclusion}

We have introduced the 3D shape reconstruction of a human body using multiple viewpoints. Whole human body data is obtained from 12 viewpoints, which is found a little occlusion. Then proposed morphable model is adapted to the whole human body data. A person swing effect is reduced thanks to high-speed 


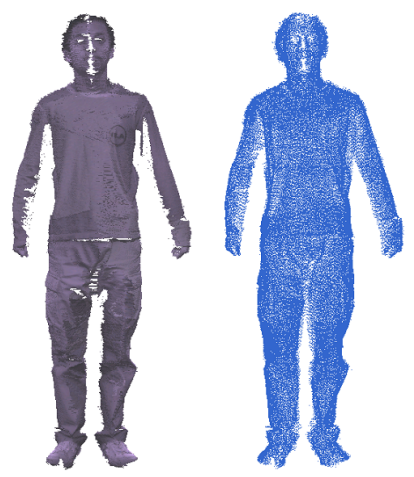

front

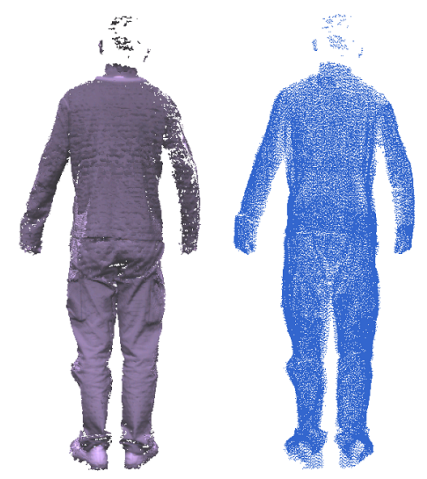

back

Fig. 11. Measurement result of a clothed male

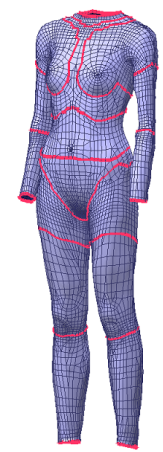

Fig. 12. Modeling result of a female

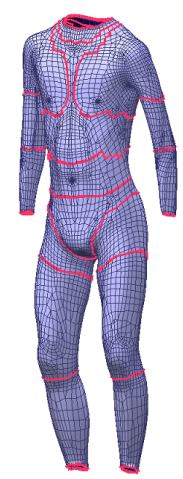

Fig. 13. Modeling result of a male

measurement in 2 seconds. The average error $1.88 \mathrm{~mm}$ is within 0.2 percent of the distance to a target. Unlike other human measurement systems, our system is configured with three poles in compact space. The assignment of pole units is not fixed and able to move itself flexibly because the pole unit is independent and movable. When we place pole units in different installation location, it is necessary to execute calibration. Promoting a rationalization of calibration is challenge that lies ahead.

Increasing the number of pole units improves the accuracy and resolves the presence of a little occlusion. Adversely if there is a good solution to complement non measurement regions of whole human body data with traditional sculptured surface or some other techniques, it is sufficient to configure with two pole units. Our approach is a flexible strategy for every situation.

Acknowledgments. This work is supported in part by a Grant-in-Aid for the Global Center of Excellence for High-Level Global Cooperation for Leading- 
Edge Platform on Access Spaces from the Ministry of Education, Culture, Sport, Science, and Technology in Japan.

\section{References}

1. Cyberware: Whole body color 3d scanner, http://www.cyberware.com/

2. VITRONIC: Vitus 3d body scanner, http://www.vitronic.de/

3. Treleaven, P.: Sizing us up. IEEE Spectrum 41, 28-31 (2004)

4. Kanade, T., Saito, H., Vedula, S.: The 3d room: Digitizing time-varying 3d events by synchronized multiple video streams. Tech. rep. CMU-RI-TR-98-34, Robotics Institute, Carnegie Mellon University (1998)

5. Saito, H., Baba, S., Kanade, T.: Appearance-based virtual view generation from multicamera videos captured in the 3-d room. IEEE Trans. Multimedia 5(3), 303316 (2003)

6. Vedula, S., Baker, S., Kanade, T.: Image-based spatio-temporal modeling and view interpolation of dynamic events. ACM Trans. Graphics 24(2), 240-261 (2005)

7. SPACEVISION: Handy 3d camera cartesia, http://www.space-vision.jp/

8. Hattori, K., Sato, Y.: Accurate rangefinder with laser pattern shifting. In: Proc. International Conference on Pattern Recognition, vol. 3, pp. 849-853 (1996)

9. Sato, Y., Otsuki, M.: Three-dimensional shape reconstruction by active rangefinder. In: IEEE Conf. Computer Vision and Pattern Recognition, pp. 142 147. IEEE Computer Society Press, Los Alamitos (1993)

10. Tsai, R.: A versatile camera calibration technique for high-accuracy $3 \mathrm{~d}$ machine vision metrology using off-the-shelf tv cameras and lens. IEEE Journal of Robotics and Automation 3(4), 323-344 (1987)

11. Koller, D., Turitzin, M., Levoy, M., Tarini, M., Croccia, G., Cignoni, P., Scopigno, R.: Protected interactive $3 \mathrm{~d}$ graphics via remote rendering. IEEE Journal of Robotics and Automation 23(3), 695-703 (2004)

12. Koller, D., Levoy, M.: Protecting 3d graphics content. Communications of the ACM 48(6), 74-80 (2005)

13. Allen, B., Curless, B., Popovi, Z.: The space of all body shapes: Reconstruction and parameterization from range scans. ACM trans. on Graphics 22(3), 587-594 (2003)

14. Allen, B., Curless, B., Popovi, Z., Hertzmann, A.: Learning a correlated model of identity and pose-dependent body shape variation for real-time synthesis. In: Proc. of the 2006 ACM SIGGRAPH/Eurographics symposium on Computer animation, vol. 48, pp. 147-156 (2006)

15. E frontier: Poser, http://www.e-frontier.com/

16. Miyazaki, S., Hasegawa, J., Yasuda, T., Yokoi, S.: A deformable object model for virtual manipulation based on maintaining local shapes. In: Proc. World MultiConference on Systemics, Cybernetics and Informatics, vol. 6, pp. 100-105 (2001)

17. Kameshima, H., Sato, Y.: Interactive adaptation for 3-d human body model to range data. In: Proc. SICE-ICASE International Joint Conference (20020061), pp. 3523-3526. 